\title{
Intratecal administration of nicotinic agonists attenuates pain-related behavior in a rat model of trigeminal neuropathic pain
}

\author{
Kunihiro Nakaia, Aya Nakae ${ }^{b, d}$, Tateki Kuboc, Yoshiki Minegishia, Yuji Fujinod, Ko Hosokawac \\ aUniversity of Fukui Hospital, Department of Plastic \& Reconstructive Surgery, Eiheiji-cho, Japan \\ bOsaka University Graduate School of Frontier Bioscience, Special Research Promotion Group, Suita Japan \\ 'Osaka Unversity Graduate School of Medicine, Department of Plastic Surgery, Suita, Japan \\ dOsaka University Graduate School of Medicine, Department of Anesthesiology \& Intensive Care Medicine, Suita, Japan
}

\section{Background and Goal of Study}

Nicotinic acetylcholine receptors (nAChRs) are widely expressed in the spinal cord and modulate nociceptive transmission. Spinal administration of the nicotinic agonists has been shown to produce antinociception. The contribution of spinal nAChRs in inhibiting the trigeminal neuropathic pain remained unclear. Chronic constriction injury to the infraorbital nerve (ION-CCI) has proven a useful model for trigeminal neuropathic pain. The present study evaluated the possible role of spinal nAChRs in ION-CCI rat model.

\section{Materials and Methods}

\section{Surgical procedures}

Male Sprague Dawley rats underwent unilateral $\mathrm{CCl}$ to the right ION. Two nylon (5-0) ligatures were tied around the ION. A polyethylene (PE-10) catheter was implanted for upper cervical spinal injection of drugs on day 14 after surgery.

\section{Drug testing}

The potential anti-allodynic effects from intrathecal administration of a nAChR agonist nicotine and an alpha 7 nAChR agonist PNU 282987 were examined. The role of nAChR and alpha $7 \mathrm{nAChR}$ in these effects was assessed by determine whether the anti-allodynic effects were prevented by intrathecal administration of a nAChR antagonist mecamylamine or an alpha $7 \mathrm{nAChR}$ antagonist methyllycaconitine.

Series of von Frey filaments were used to determine pain hypersensitivity to mechanical stimulation. Withdrawal thresholds were measured before and, 30 , 60, 90 and 120 min after drug injection.

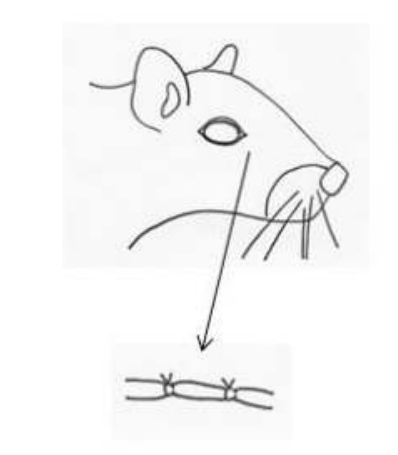

Chronic constriction injury to the infraorbital nerve $(\mathrm{ION}-\mathrm{CCl})$

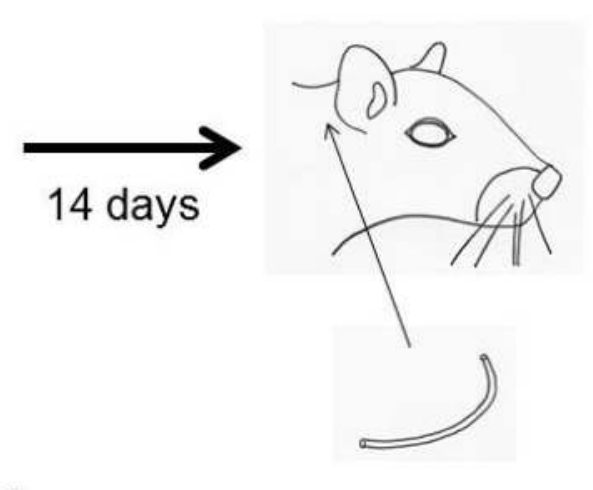

Implantation of a polyethylene catheter

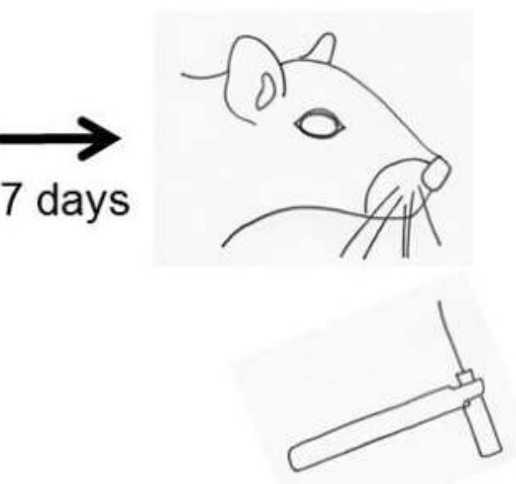

Intrathecal administration and applicaition of von Frey filaments

Figure 1 Experimental schedule

\section{Results and Discussion}

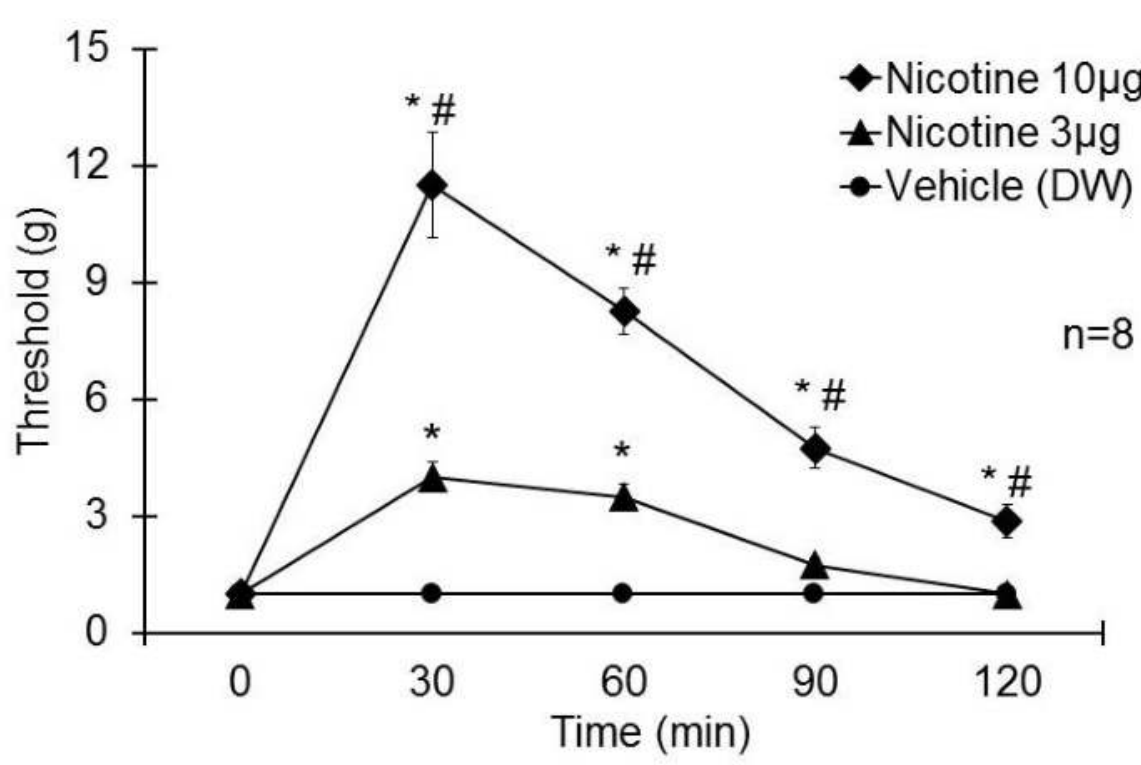

Figure 2 Intrathecal administration of nicotine (nAChR agonist) produced dose-dependent antiallodynic effects. $(n=8)$ ${ }^{*} \mathrm{P}<0.05$ compared with vehicle (DW) group. $\# \mathrm{P}<0.05$ compared with $3 \mu \mathrm{g}$-treated group.

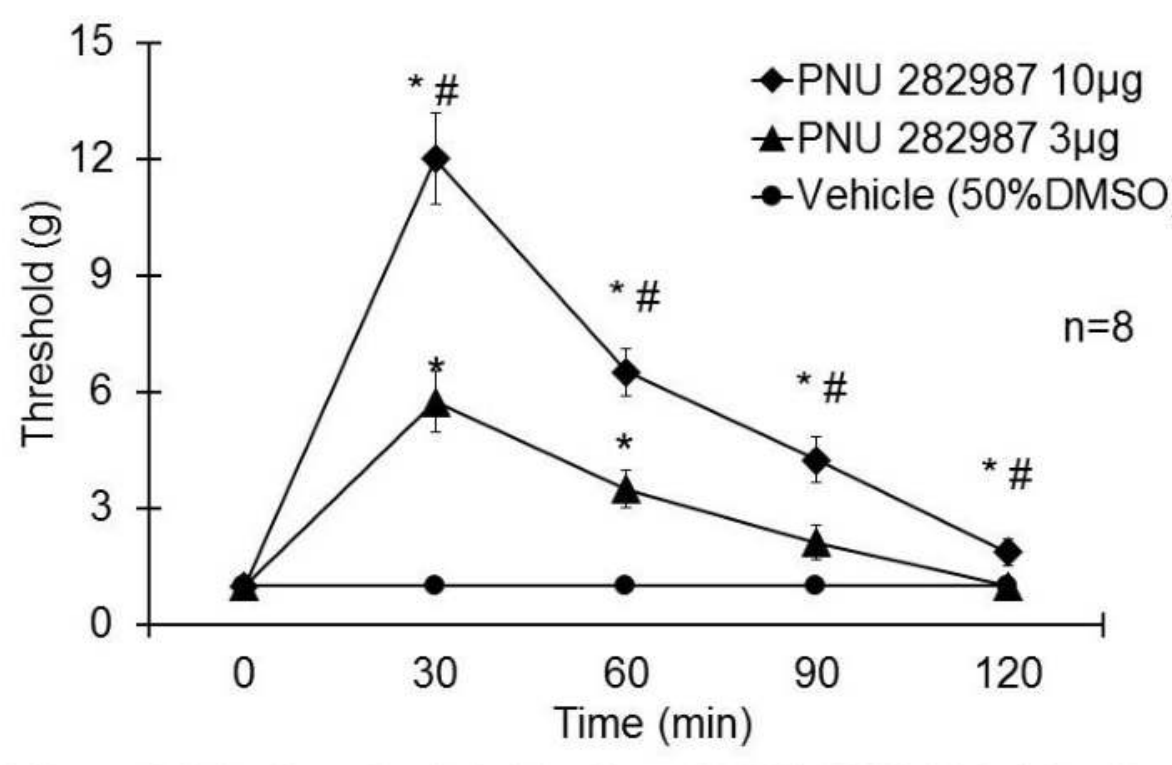

Figure 4 Intrathecal administration of PNU 282987 (alpha 7 nAChR agonist) produced dose-dependent antiallodynic effects. $(n=8)$

${ }^{*} \mathrm{P}<0.05$ compared with vehicle (DW) group. $\# \mathrm{P}<0.05$ compared with $3 \mu \mathrm{g}$-treated group.

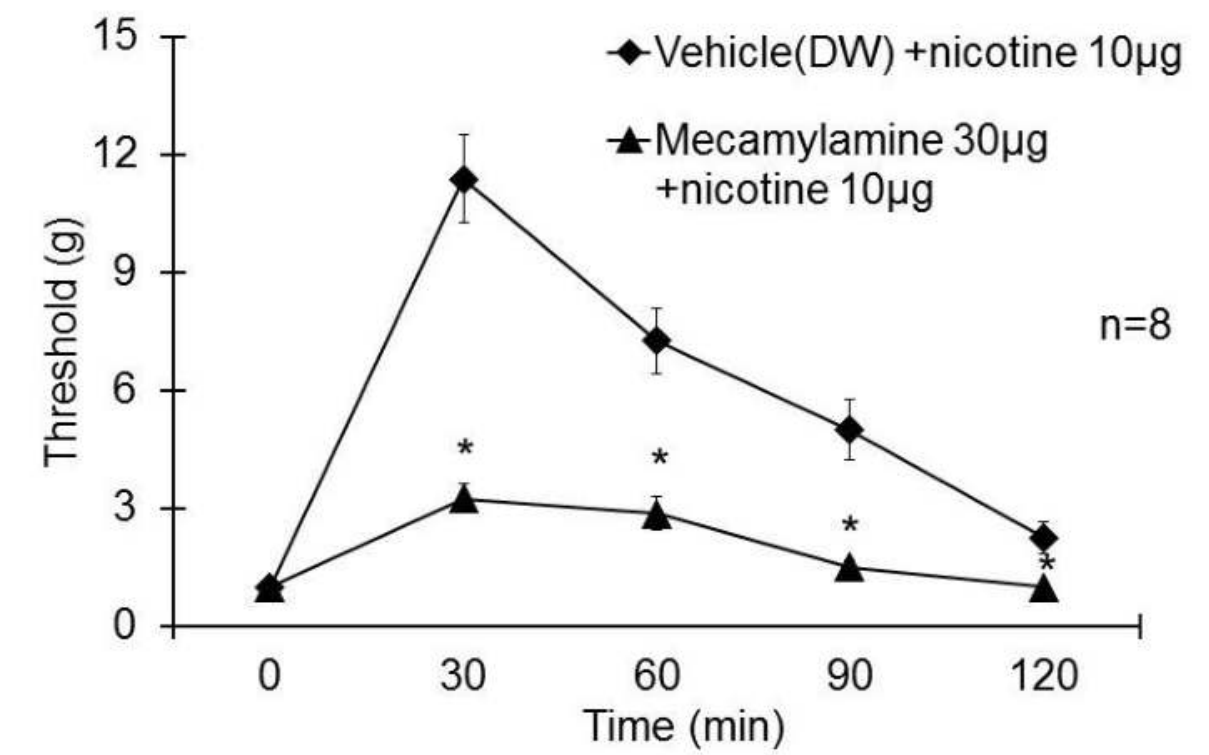

Figure 3 The anti-allodynic effect of nicotine $10 \mu \mathrm{g}(\mathrm{nAChR}$ agonist) was significantly reduced by mecamylamine $30 \mu \mathrm{g}$ (nAChR antagonist). ( $n=8)$

${ }^{\star} \mathrm{P}<0.05$ compared with vehicle $(D W)+$ nicotine $10 \mu \mathrm{g}$ group.

\section{Conclusion}

The results indicated that spinal nAChRs and alpha 7 nAChRs may play an important role in a rat model of trigeminal neuropathic pain.

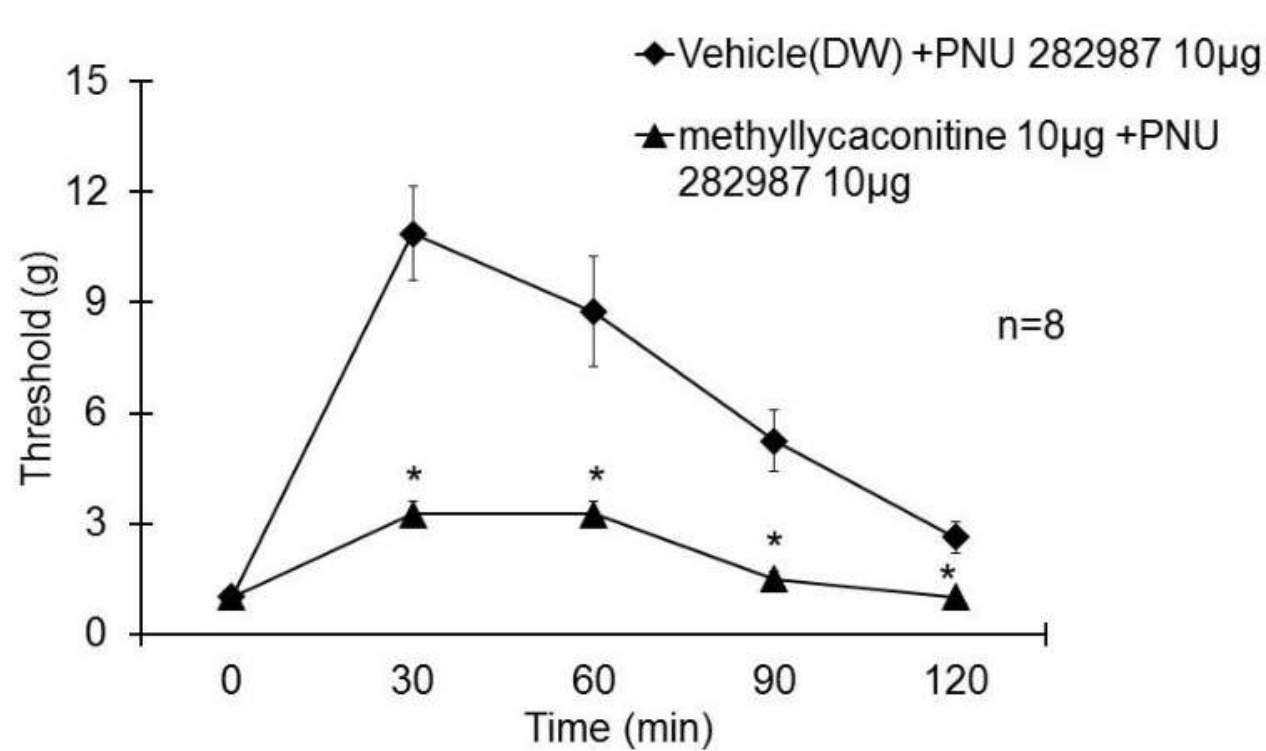

Figure 5 The anti-allodynic effect of PNU $28298710 \mu \mathrm{g}$ (alpha $7 \mathrm{nAChR}$ agonist) was significantly reduced by methyllycaconitine $10 \mu \mathrm{g}$ (alpha 7 $\mathrm{nAChR}$ antagonist). $(\mathrm{n}=8)$

${ }^{*} \mathrm{P}<0.05$ compared with vehicle (DW) + PNU $28298710 \mu \mathrm{g}$ group.

\section{Acknowledgements}

